\title{
ARTICLE OPEN \\ Improving the gate fidelity of capacitively coupled spin qubits
}

\author{
Xin Wang ${ }^{1,3}$, Edwin Barnes ${ }^{1,2}$ and S Das Sarma ${ }^{1,2}$
}

Capacitively coupled semiconductor spin qubits hold promise as the building blocks of a scalable quantum computing architecture with long-range coupling between distant qubits. However, the two-qubit gate fidelities achieved in experiments to date have been severely limited by decoherence originating from charge noise and hyperfine interactions with nuclear spins, and are currently unacceptably low for any conceivable multi-qubit gate operations. Here, we present control protocols that implement two-qubit entangling gates while substantially suppressing errors due to both types of noise. These protocols are obtained by making simple modifications to control sequences already used in the laboratory and should thus be easy enough for immediate experimental realisation. Together with existing control protocols for robust single-qubit gates, our results constitute an important step toward scalable quantum computation using spin qubits in semiconductor platforms.

npj Quantum Information (2015) 1, 15003; doi:10.1038/npjqi.2015.3; published online 27 October 2015

\section{INTRODUCTION}

Precise manipulation of coupled quantum systems is at the heart of quantum technologies. Quantum gates acting on two or more qubits are the building blocks of quantum algorithms and the engines of entanglement creation, the key feature which sets apart many technological applications such as quantum information processing and quantum communication ${ }^{1}$ from their classical counterparts. The ability to manipulate two or more interacting qubits has been demonstrated with reasonably high fidelity in many systems, such as superconducting qubits, ${ }^{2}$ trapped ions ${ }^{3}$ and optical systems, ${ }^{4}$ but for the purpose of quantum computing, scaling to many qubits may be ultimately easier to achieve with semiconductor spin qubits ${ }^{5-9}$ because they are nanoscale devices that can potentially take advantage of the preexisting infrastructure for fabricating semiconductor microchips. ${ }^{10}$ The singlettriplet qubit, ${ }^{11}$ which encodes a qubit in two-electron spin states, bears the advantage that it is immune to homogeneous fluctuations in the external magnetic field and is the simplest spin qubit that can be controlled purely electrically. Although it has had vast success at the single-qubit level with long coherence times and high control fidelities, ${ }^{12-14}$ the implementation of two-qubit gates has been challenging and is currently the bottleneck preventing progress towards demonstrations of quantum algorithms, which necessitate both single- and multiqubit gate operations.

Attempts to design high-fidelity two-qubit gates for singlettriplet qubits have mostly assumed that the qubits are coupled via the exchange interaction, ${ }^{15,16}$ in which case one may focus on one pair of exchange-coupled spins at a time, a great simplification from the original four-spin problem. Despite its theoretical simplicity and elegance, this approach is hard to implement in experiments with multiple gate-defined quantum dots due to difficulties in addressing a single exchange coupling in the array. An alternative proposal couples two singlet-triplet qubits with a capacitive interaction, that is, one may alter the electrostatic potential, and hence the precession rate, in the 'target' qubit by changing the electron charge configuration of the 'control' qubit. $^{10,17}$ This proposal allows for long-range, individually controllable couplings between qubits, ${ }^{18}$ which is important for multi-qubit devices, but also has a serious difficulty in that the capacitive coupling is typically much weaker than the exchange coupling and is much more susceptible to noise. An experimental breakthrough in this problem came from the use of a 'simultaneous dynamical decoupling' sequence which cancels part of the noise-induced evolution error, leading to the first demonstration of a singlet-triplet two-qubit gate, with a fidelity of $72 \%{ }^{19}$ To meet the requirement for fault-tolerant quantum computation, one must further reduce the error either at the hardware level by enhancing the capacitive coupling or making better noise-free samples, or at the software level using dynamically corrected gates. Several theoretical works have used the latter approach to design higher-fidelity single-qubit gates. ${ }^{16,20-23}$

Despite the experimental advance demonstrated in reference 19 , theoretical research on improving the fidelity of singlet-triplet two-qubit gates via capacitive coupling has remained rare. Although the inter-qubit coupling term has been individually treated in $\mathrm{NMR}^{24}$ the unavoidable presence of single-qubit terms and their associated errors complicates the problem considerably, making previous approaches inapplicable. Further difficulty arises in which capacitive coupling exhibits less symmetry compared with the exchange coupling, preventing one from factorising the larger two-qubit Hilbert space into separate subspaces. This makes it difficult to adapt (or generalise) dynamically corrected gates developed for single-qubit operations ${ }^{16,20-23}$ to design a robust two-qubit gate for capacitive coupling as was done in the case of exchange coupling. ${ }^{16,22}$ Still, it has recently been realized that one may systematically generate entangling gates with capacitive coupling, which are equivalent to the well-known CNOT gate by using a single square pulse. ${ }^{25}$ In addition, the decoherence mechanisms relevant for the protocol implemented in reference 19 have been further investigated in reference 26 . However, a

${ }^{1}$ Condensed Matter Theory Center, Department of Physics, University of Maryland, College Park, MD, USA and ${ }^{2}$ Joint Quantum Institute, University of Maryland, College Park, MD, USA.

Correspondence: X Wang (x.wang@cityu.edu.hk)

${ }^{3}$ Current address: Department of Physics and Materials Science, City University of Hong Kong, Hong Kong SAR, China

Received 24 December 2014; revised 15 April 2015; accepted 4 May 2015 
systematic way of performing two-qubit gate operations that are robust against noise is still an important open question. Here, we address this issue by showing that minor, precise modifications to the sequence already experimentally implemented in reference 19 can further cancel the effects of noise and significantly enhance the two-qubit gate fidelity. We start with the simpler case in which the magnetic field gradient is much smaller than the control field; using the results we obtain in this limit as a guide, we then solve the problem for more general parameter regimes. We show how to systematically generate pulse sequences that implement entangling two-qubit gates while suppressing both nuclear and charge noise errors by more than an order of magnitude.

\section{MATERIALS AND METHODS}

We consider two singlet-triplet qubits (labelled by $A$ and $B$ ) coupled capacitively. The Hamiltonian reads ${ }^{19}$

$$
\begin{aligned}
H\left(\left\{J^{A}, h^{A}\right\},\left\{J^{B}, h^{B}\right\}, J^{A B}\right)= & \left(J^{A} \sigma_{z}+h^{A} \sigma_{x}\right) \otimes I+I \otimes\left(J^{B} \sigma_{z}+h^{B} \sigma_{x}\right) \\
& +J^{A B} \sigma_{z} \otimes \sigma_{z} .
\end{aligned}
$$

Here, $J^{A}\left(J^{B}\right)$ is the exchange interaction between the two spins on qubit $A$ $(B)$ and determines the rate of single-qubit $z$-rotations. $h^{A}\left(h^{B}\right)$ is the magnetic field gradient enabling single-qubit $x$-rotations. $f^{A B}$ denotes the capacitive coupling between the two qubits which creates entanglement, and is typically much smaller than $J^{A}$ and $J^{B}$. In practice, the exchange interactions are controlled by the bias voltages (detunings) applied to each qubit, $J^{A}=J^{A}\left(\varepsilon^{A}\right)$ and $J^{B}=J^{B}\left(\varepsilon^{B}\right)$. One can similarly write $J^{A B}=J^{A B}\left(\varepsilon^{A B}\right)$, where $\varepsilon^{A B}$ may be defined as a linear combination of $\varepsilon^{A}$ and $\varepsilon^{B}$. The evolution under the Hamiltonian, equation (1), for time $t$ is thus a two-qubit gate denoted as:

$$
U\left(\left\{J^{A}, h^{A}\right\},\left\{J^{B}, h^{B}\right\}, J^{A B}, t\right)=e^{-i H\left(\left\{J^{A}, h^{A}\right\},\left\{J^{B}, h^{B}\right\},,^{A B}\right) t},
$$

and may be expressed analytically as:

$$
\begin{aligned}
U= & \sum_{k=1}^{4} \frac{e^{r_{k} t}}{4 A_{k}}\left(A_{k} I \otimes I+u_{i x, k} I \otimes \sigma_{x}+u_{x i, k} \sigma_{x} \otimes I+u_{i z, k} I \otimes \sigma_{z}+u_{z i, k} \sigma_{z} \otimes I\right. \\
& +u_{x z, k} \sigma_{x} \otimes \sigma_{z}+u_{z x, k} \sigma_{z} \otimes \sigma_{x}+u_{x x, k} \sigma_{x} \otimes \sigma_{x} \\
& \left.+u_{y y, k} \sigma_{y} \otimes \sigma_{y}+u_{z z, k} \sigma_{z} \otimes \sigma_{z}\right)
\end{aligned}
$$

where we have defined

$$
\begin{aligned}
& A_{k}=\left[\left(h^{A}\right)^{2}+\left(h^{B}\right)^{2}+\left(J^{A}\right)^{2}+\left(J^{B}\right)^{2}+\left(J^{A B}\right)^{2}\right] r_{k}-2 i J^{A} J^{B} J^{A B}+r_{k}^{3}, \\
& u_{i x, k}=-i h^{B}\left[-\left(h^{A}\right)^{2}+\left(h^{B}\right)^{2}-\left(J^{A}\right)^{2}+\left(J^{B}\right)^{2}+\left(J^{A B}\right)^{2}+r_{k}^{2}\right], \\
& u_{x i, k}=u_{i x, k}\left(J^{A} \leftrightarrow J^{B}, h^{A} \leftrightarrow h^{B}\right), \\
& u_{i z, k}=-i\left\{-J^{B}\left[\left(h^{A}\right)^{2}-\left(h^{B}\right)^{2}+\left(J^{A}\right)^{2}+\left(J^{A B}\right)^{2}\right]+J^{B} r_{k}^{2}-2 i J^{A} J^{A B} r_{k}+\left(J^{B}\right)^{3}\right\}, \\
& u_{z i, k}=u_{i z, k}\left(J^{A} \leftrightarrow J^{B}, h^{A} \leftrightarrow h^{B}\right), \\
& u_{x z, k}=2 i h^{A}\left(J^{A} J^{A B}+i J^{B} r_{k}\right), \\
& u_{z x, k}=u_{x z, k}\left(J^{A} \leftrightarrow J^{B}, h^{A} \leftrightarrow h^{B}\right), \\
& u_{x x, k}=-2 h^{A} h^{B} r_{k}, \\
& u_{y y, k}=-2 i h^{A} h^{B} J^{A B}, \\
& u_{z z, k}=-i\left\{J^{A B}\left[\left(h^{A}\right)^{2}+\left(h^{B}\right)^{2}-\left(J^{A}\right)^{2}-\left(J^{B}\right)^{2}+\left(J^{A B}\right)^{2}\right]+J^{A B} r_{k}^{2}-2 i J^{A} J^{B} r_{k}\right\} .
\end{aligned}
$$

The $r_{k}$ are the roots of the following polynomial:

$$
P(r)=a+b r+c r^{2}+r^{4},
$$

with

$$
\begin{aligned}
& a=2\left(J^{A B}\right)^{2}\left[\left(h^{A}\right)^{2}+\left(h^{B}\right)^{2}-\left(J^{A}\right)^{2}-\left(J^{B}\right)^{2}\right] \\
& +\left[\left(h^{A}\right)^{2}-\left(h^{B}\right)^{2}+\left(J^{A}\right)^{2}-\left(J^{B}\right)^{2}\right]^{2}+\left(J^{A B}\right)^{4}, \\
& b=-8 i J^{A} J^{B} A^{A B}, \\
& c=2\left[\left(h^{A}\right)^{2}+\left(h^{B}\right)^{2}+\left(J^{A}\right)^{2}+\left(J^{B}\right)^{2}+\left(J^{A B}\right)^{2}\right] .
\end{aligned}
$$

Similar to the single-qubit case, the evolution of two qubits is subject to two noise channels. In the case where the qubits are in semiconductors like $\mathrm{GaAs},{ }^{13,27,28}$ the nuclear noise arises from the flip-flops of surrounding nuclear spins mediated by the hyperfine interaction with the electron spins in the quantum dots, which changes $h^{A}$ to $h^{A}+\delta h^{A}$ and $h^{B}$ to $h^{B}+\delta h^{B} .{ }^{29}$ On the other hand, charged impurities near the confined electrons lead to fluctuations of the confinement potential and consequently the exchange interaction. The shift in the exchange interaction can be expressed as $j^{A} \rightarrow J^{A}+g^{A}\left(J^{A}\right) \delta \varepsilon^{A}, j^{B} \rightarrow J^{B}+g^{B}\left(J^{B}\right) \delta \varepsilon^{B}$ and $j^{A B} \rightarrow A^{A B}+g^{A B}\left(J^{A}\right) \delta \varepsilon^{A B}$, where $g$ is determined by the dependence of $J$ on the detuning $\varepsilon .^{30}$ Together, these errors give rise to inaccuracies in the resulting two-qubit quantum gates, namely $U \rightarrow U+\delta U$ with $\delta U$ dependent on the $\delta h^{\prime}$ s and $\delta \varepsilon^{\prime}$ s. The goal of this paper is to reduce the effect of noise (i.e., $\delta U$ ) using optimised pulse sequences. In this work, we also make the static-noise approximation, namely the $\delta h^{\prime}$ 's and $\delta \varepsilon^{\prime}$ s are assumed to be unknown constants for a given run of a quantum gate, but may change from one run to the next. The static-noise approximation is valid in most experimental spin qubit situations since the electrically controlled gate operations can typically be very fast compared with the slow noise fluctuations in the environment. ${ }^{28,30}$ For spin qubits in silicon, the nuclear noise is absent and one has only charge noise to deal with. ${ }^{31-33}$ Our results are applicable in this case as well.

The explicit analytical form of equation (2) for arbitrary parameters is complicated and involves solutions to a quartic eigenequation, making it difficult to find pulse sequences which implement well-known entangling gates such as the CNOT. In reference 19, a two-qubit gate was experimentally demonstrated by using a 'simultaneous dynamical decoupling' sequence given by

$$
\begin{aligned}
& U\left(\left\{J^{A}, 0\right\},\left\{J^{B}, 0\right\}, J^{A B}, t\right) e^{-i \frac{\pi}{2}\left(\mid \otimes \sigma_{x}+\sigma_{x} \otimes l\right)} U\left(\left\{J^{A}, 0\right\},\left\{J^{B}, 0\right\}, J^{A B}, t\right) \\
& \quad=\left(\begin{array}{llll}
0 & 0 & 0 & -e^{-i \phi} \\
0 & 0 & -e^{i \phi} & 0 \\
0 & -e^{i \phi} & 0 & 0 \\
-e^{-i \phi} & 0 & 0 & 0
\end{array}\right),
\end{aligned}
$$

where $\phi=2\lrcorner^{A B} t$, and one makes the approximation that $h^{A}$ and $h^{B}$ are much weaker compared with the control fields and are thus negligible when those fields are pulsed on. The resulting gate has a particularly useful anti-diagonal form. One typically uses the Makhlin invariants ${ }^{34}$ to classify two-qubit gates such that two gates with identical invariants may be converted into each other using only single-qubit (local) operations. The Makhlin invariants for equation (7) are $G_{1}=\cos ^{2} 2 \phi$ and $G_{2}=2+\cos 4 \phi$. For $\phi=N \pi / 4$ with $N$ an odd integer, it is equivalent to CNOT $\left(G_{1}=0, G_{2}=1\right)$ up to local operations.

\section{RESULTS}

In this paper, we start from equation (7) and show how one can reduce the gate error due to nuclear noise and charge noise. For the first half of our results, we make the same assumption $h^{A}$ $h^{B} \ll J^{A}, J^{B}, J^{A B}$ that has been utilised and verified in experiments. ${ }^{19}$ In this relatively simple case, we show that one may construct dynamically corrected two-qubit gates in an intuitive way. Later on, we discuss the more complicated, yet practical, case with nonzero $h^{A}$ and $h^{B}$, where it is harder to gain intuition even in the absence of noise, and extra work is needed to ensure the equivalence between the outcome of the pulse sequence and the desired two-qubit gate (CNOT) by direct evaluation of the Makhlin invariants. Nevertheless, we explicitly demonstrate that pulse sequences can be constructed in this demanding situation as well using constrained numerical optimisation techniques that start from the solution to the simpler $h^{A}=h^{B}=0$ case.

We also make a few nonessential assumptions for the purpose of presentation. We show results for 'symmetric' pulsing, namely $J^{A}=J^{B}=J$ and $h^{A}=h^{B}=h$. Regarding the charge noise, we assume the empirical form ${ }^{30} J^{A}=J_{0}^{A} \exp \left(\varepsilon^{A} / \varepsilon_{0}\right)$ so that $\delta J^{A}=J^{A} \delta \varepsilon^{A} / \varepsilon_{0}$ and similarly $\delta J^{B}=J^{B} \delta \varepsilon^{B} / \varepsilon_{0}$. For the capacitive coupling $J^{A B}$, the mechanism of charge noise is different than for individual qubits. Although in reference 19 it has been argued empirically that $J^{A B} \propto J^{A} J^{B}$, no microscopic calculation of this relationship has appeared in the literature, and the precise form is likely sample-dependent. We therefore assume the simple form $\delta J^{A B}=J^{A B} \delta \varepsilon^{A B} / \varepsilon_{0}$. Although we make these assumptions because concrete parameter values are needed in evaluating the performance of the gates, we emphasise that the applicability of our method does not depend in any way on these assumptions. In 
fact, all these restrictions can be lifted either by modifying the cost function or altering the parameters used in the numerical search. For example, deviations from the assumed exponential model of charge noise may easily be accommodated according to the method presented in reference 22 .

Nuclear noise reduction for $h=0$ case

We write the evolution operator, equation (7), under nuclear noise $\delta h^{A}$ and $\delta h^{B}$ as

$$
\begin{aligned}
\tilde{F}\left(J, h, \phi, \delta h^{A}, \delta h^{B}\right)= & U\left(\left\{J, h+\delta h^{A}\right\},\left\{J, h+\delta h^{B}\right\}, J^{A B},\right. \\
& \left.\phi / J^{A B}\right) e^{-i \frac{\pi}{2}\left(\mid \otimes \sigma_{x}+\sigma_{x} \otimes l\right)} \times U\left(\left\{J, h+\delta h^{A}\right\},\right. \\
& \left.\left\{J, h+\delta h^{B}\right\}, J^{A B}, \phi / J^{A B}\right) .
\end{aligned}
$$

We also define the noise-free component of equation (8) as $F(J, h, \phi)=\tilde{F}(J, h, \phi, 0,0)$, shown as the first quantum circuit in Figure 1a. For $h=0$, the expansion of equation (8) to first order in $\delta h$ only contains terms proportional to $\sigma_{x} \otimes I, \sigma_{x} \otimes \sigma_{z \prime}, l \otimes \sigma_{x}$ and $\sigma_{z} \otimes \sigma_{x}$ as is clear from the Materials and Methods section. For this particular case, the explicit form of these error terms is simple enough to be obtained analytically. We write the first-order error terms as $(\zeta=A, B)$

$$
\begin{aligned}
\frac{\partial \tilde{F}\left(J, 0, \phi, \delta h^{A}, \delta h^{B}\right)}{\partial \delta h^{\zeta}}= & f_{x 0}^{\zeta} \sigma_{x} \otimes I+f_{x z}^{\zeta} \sigma_{x} \otimes \sigma_{z}+f_{0 x}^{\zeta} I \otimes \sigma_{x} \\
& +f_{z x}^{\zeta} \sigma_{z} \otimes \sigma_{x},
\end{aligned}
$$

and for a given $\phi$ value, the coefficients on the right-hand side of equation (13) are only functions of $J / J^{A B}$. As it is easy to vary $J$ experimentally, it makes sense to choose a value that minimises the error. Therefore, we reiterate the problem as a constrained optimisation problem: given the cost function $K(J)=\sum_{\zeta=A, B}\left(\left|f_{x 0}^{\zeta}\right|^{2}+\left|f_{x z}^{\zeta}\right|^{2}+\left|f_{0 x}^{\zeta}\right|^{2}+\left|f_{z x}^{\zeta}\right|^{2}\right)$ and the constraint that $J \geqslant J^{A B}$ (which is the physical regime where experiments operate ${ }^{19}$ ), find the value of $J$ which minimises $K(J)$. To simplify expressions of cost functions to be discussed later, we define the 'norm' of a $4 \times 4$ matrix $Q$ projected onto the 16-dimensional two-qubit Pauli basis as $\|Q\|=\left[\sum_{\mu, \nu}\left|\operatorname{Tr} Q\left(\sigma_{\mu} \otimes \sigma_{\nu}\right) / 4\right|^{2}\right]^{1 / 2}$ and $\mu, v$ run over $\{0, x, y, z\}$ while $\sigma_{0}=l$. Then

$$
K(J)=\sum_{\zeta=A, B}\left\|\partial \tilde{F} / \partial \delta h^{\zeta}\right\|^{2} .
$$

Here, we use the norm of the 'error vector' in place of the infidelity because the latter requires input of the noise amplitude, which we assume to be an unknown constant for a given run.

We show $K(J)$ in Figure 1b. $K(J)$ has multiple minima, and one can choose the minimum which is most practical for a specific experiment. As the curve does not have sharp dips at the minima, even if one is a little off from the optimal value, one should still expect error reduction. Results for $J=9.2901 \mathcal{A}^{A B}$ are shown in Figure $1 \mathrm{c}$ as the red dashed line, which is compared with an unoptimized (arbitrarily chosen) value of $J=3 J^{A B}$ for the sequence of equation (8). (All curves shown in Figure 1c correspond to $\phi=3 \pi / 4$ and are equivalent to CNOT, and, for presentational purposes, we have set $\delta h^{A}=\delta h^{B}=\delta h$.) Because there is only one instance of the evolution operator $U$ appearing on each side of the $x$-rotations, we term the sequence as 'level- 1 '. We see that if one simply changes $J$ to the value $9.2901 J^{A B}$, the sequence already offers an additional error reduction by two orders of magnitude. Even if we cannot cancel the first-order $\delta h$ error entirely, as is evident from the equal slopes of the lines in Figure 1c, this reduction in error is already substantial, and it only requires a simple alteration in existing experiments (just changing the $J$ value). Here we also note that if one allows asymmetric pulsing (i.e., $J^{A} \neq J^{B}$ ), the cost function is instead simply $K\left(J^{A}, J^{B}\right)=\left[K\left(J^{A}\right)+K\left(J^{B}\right)\right] / 2$, where $K(J)$ is as defined in equation (10). The minima are therefore the same as those for equation (10). We also note that although $f^{A B}$ is assumed to be held constant in producing the figure, our method can easily be adapted to more general situations that can include nontrivial relations between $\mathcal{J}^{A}, J^{B}$ and $J^{A B}$.

a

$$
\begin{aligned}
& A-U\left(\{J, h\},\{J, h\}, J^{A B}, t\right)-R(\hat{x}, \pi) \\
& R(\hat{x}, \pi)
\end{aligned}\left(\{J, h\},\{J, h\}, J^{A B}, t\right) \equiv F\left(J, h, \phi=2 J^{A B} t\right)
$$

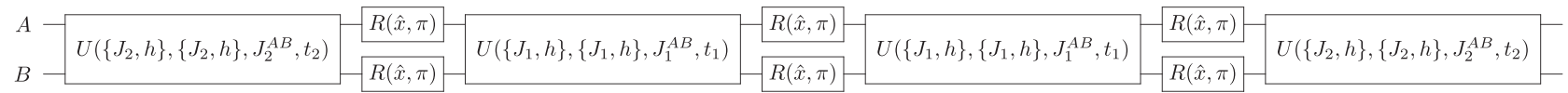

\section{b}

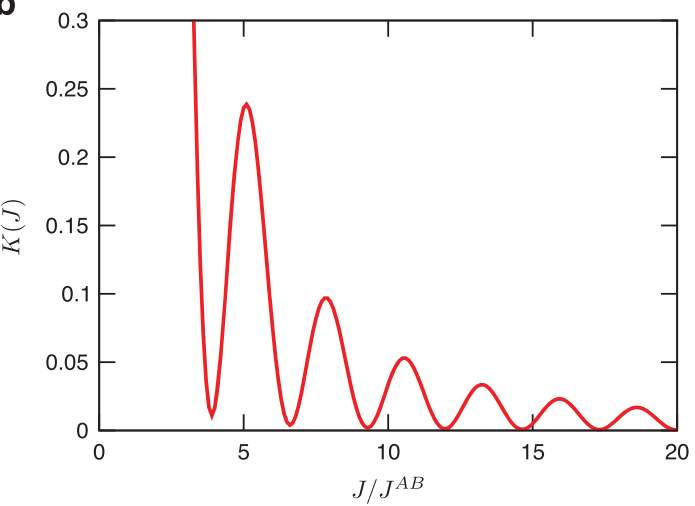

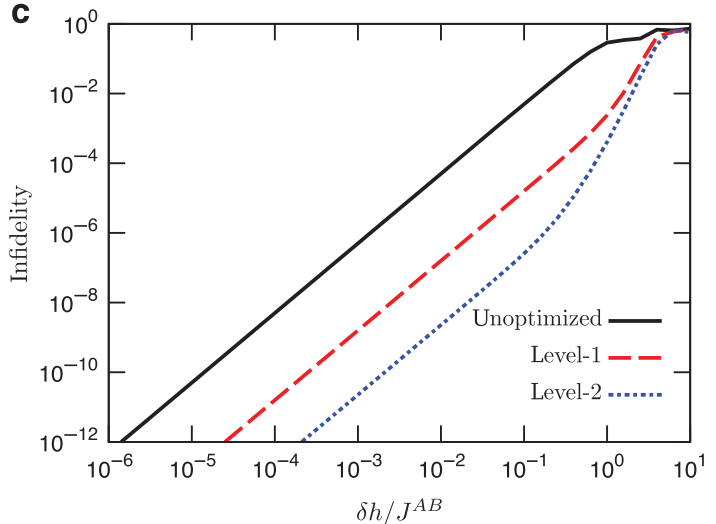

Figure 1. Nuclear noise reduction for $h=0$. The charge noise has been set to zero. (a) Quantum circuits showing the level-1 and level-2 pulse sequences used to reduce the nuclear noise. (b) The cost function, equation (10). (c) Infidelity versus nuclear noise $\delta h / J^{A B}$ for unoptimized (black line), level-1 optimised (red dashed line) and level-2 optimised (blue dotted line) sequences. Parameters: unoptimized pulse: $J=3 J^{A B}$, $\phi=3 \pi / 4\left[t=3 \pi / 8 J^{A B}\right]$; optimised one-piece pulse: $J=9.2901 J^{A B}, \phi=3 \pi / 4\left[t=3 \pi /\left(8 \mathcal{A}^{A B}\right)\right]$; optimised two-piece pulse: $J_{1}=18.767 J^{A B}, J_{2}=9.2123 J^{A B}{ }^{\prime}$, $t_{1}=0.33606 / J^{A B}, t_{2}=3 \pi /\left(8 J^{A B}\right)-t_{1}$. 
a
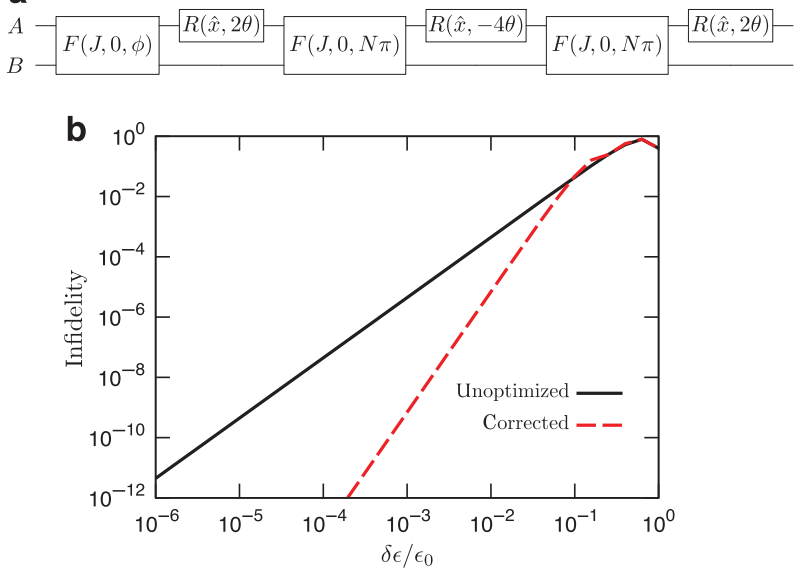

Figure 2. Charge noise reduction for $h=0$ while the nuclear noise is absent. (a) Quantum circuit showing the pulse sequence used to cancel the charge noise. (b) Infidelity versus charge noise $\delta \varepsilon / \varepsilon_{0}$ for unoptimized (black line) and corrected (red dashed line) sequences. For the unoptimized pulse, we choose $J=3 A^{A B}, \phi=3 \pi / 4$, while for the corrected pulse, we have $J=3 J^{A B}, \phi=3 \pi / 4, N=4$, $\theta=\frac{1}{2} \arccos \left(-\frac{\phi}{2 N \pi}\right)$.

Further error reduction may be achieved by adding more parameters in the optimisation scheme. We note that

$$
\begin{aligned}
& \prod_{j=n}^{1}\left[U\left(\left\{J_{j}^{A}, 0\right\},\left\{J_{j}^{B}, 0\right\}, J_{j}^{A B}, \phi_{j} /\left(2 J_{j}^{A B}\right)\right) e^{-i \frac{\pi}{2}\left(l \otimes \sigma_{x}+\sigma_{x} \otimes l\right)}\right] e^{i \frac{\pi}{2}\left(l \otimes \sigma_{x}+\sigma_{x} \otimes l\right)} \\
& \prod_{j=1}^{n}\left[e^{-i \frac{\pi}{2}\left(l \otimes \sigma_{x}+\sigma_{x} \otimes l\right)} U\left(\left\{J_{j}^{A}, 0\right\},\left\{J_{j}^{B}, 0\right\}, J_{j}^{A B}, \phi_{j} /\left(2 J_{j}^{A B}\right)\right)\right] \\
& =\left(\begin{array}{llll}
0 & 0 & 0 & -e^{-i \sum_{j} \phi_{j}} \\
0 & 0 & -e^{i \sum_{j} \phi_{j}} & 0 \\
0 & -e^{i \sum_{j} \phi_{j}} & 0 & 0 \\
-e^{-i \sum_{j} \phi_{j}} & 0 & 0 & 0
\end{array}\right)
\end{aligned}
$$

Under our assumption of symmetric dots $J_{j}^{A}=J_{j}^{B}=J_{j}$, we have two parameters $J_{1}$ and $J_{2}$ for $n=2$, which we call a 'level-2' sequence. The circuit for this case is shown in Figure 1a. Defining

$$
\begin{aligned}
& \tilde{F}^{(2)}\left(J_{1}, J_{2}, \phi_{1}, \phi_{2}, \delta h_{1}^{A}, \delta h_{1}^{B}, \delta h_{2}^{A}, \delta h_{2}^{B}\right) \\
&= U\left(\left\{J_{2}, \delta h_{2}^{A}\right\},\left\{J_{2}, \delta h_{2}^{B}\right\}, J_{2}^{A B}, \phi_{2} / J_{2}^{A B}\right) e^{-i \frac{\pi}{2}\left(l \otimes \sigma_{x}+\sigma_{x} \otimes l\right)} \\
& \times U\left(\left\{J_{1}, \delta h_{1}^{A}\right\},\left\{J_{1}, \delta h_{1}^{B}\right\}, J_{1}^{A B}, \phi_{1} / J_{1}^{A B}\right) e^{-i \frac{\pi}{2}\left(l \otimes \sigma_{x}+\sigma_{x} \otimes l\right)} \\
& \times U\left(\left\{J_{1}, \delta h_{1}^{A}\right\},\left\{J_{1}, \delta h_{1}^{B}\right\}, J_{1}^{A B}, \phi_{1} / J_{1}^{A B}\right) e^{-i \frac{\pi}{2}\left(l \otimes \sigma_{x}+\sigma_{x} \otimes l\right)} \\
& \times U\left(\left\{J_{2}, \delta h_{2}^{A}\right\},\left\{J_{2}, \delta h_{2}^{B}\right\}, J_{2}^{A B}, \phi_{2} / J_{2}^{A B}\right),
\end{aligned}
$$

the problem now is to minimize the cost function

$$
K\left(J_{1}, J_{2}, \phi_{1}, \phi_{2}\right)=\sum_{\substack{\zeta=A, B \\ j=1,2}}\left\|\frac{\partial \tilde{F}^{(2)}}{\partial \delta h_{j}^{\zeta}}\right\|^{2},
$$

subject to the constraint that $\phi_{1}+\phi_{2}=N \pi / 4, \phi_{1}, \phi_{2} \geqslant 0$ and $J_{1}$, $J_{2} \geqslant \mathcal{A B}^{A B}$. Numerical results for $N=3$ are shown in Figure 1c (as in the previous case, we have set $\delta h_{1}^{A}=\delta h_{1}^{B}=\delta h_{2}^{A}=\delta h_{2}^{B}=\delta h$ ), where we see that for a wide range of $\delta h$, one may further reduce the error by two orders of magnitude relative to the optimised level-1 sequence. Therefore, the pulse sequence is very effective in reducing the error at the cost of extending the sequence by only a factor of two.
Charge noise cancellation for $h=0$ case

As mentioned above, we assume that the charge noise shifts the inter-qubit coupling by $\delta \jmath^{A B}=J^{A B} \delta \epsilon^{A B} / \epsilon_{0}$. For the $h=0$ case, it does not matter how $J^{A}$ or $J^{B}$ responds to the charge noise in the sequence of equation (7) so long as these effects remain identical before and after the single-qubit $x$-rotations on the left-hand side of equation (7), as $J^{A}$ and $J^{B}$ do not appear on the right-hand side. (This also partially explains why equation (8) produces gates with higher fidelity than a naïve implementation of equation (1).) We therefore denote $\delta \epsilon^{A B} / \epsilon_{0} \equiv \delta \epsilon$ and rewrite the right-hand side of equation (7) under the influence of charge noise as

$$
\tilde{E}(\phi, \delta \epsilon)=\left(\begin{array}{cccc}
0 & 0 & 0 & -e^{-i(1+\delta \epsilon) \phi} \\
0 & 0 & -e^{i(1+\delta \epsilon) \phi} & 0 \\
0 & -e^{i(1+\delta \epsilon) \phi} & 0 & 0 \\
-e^{-i(1+\delta \epsilon) \phi} & 0 & 0 & 0
\end{array}\right) .
$$

This type of amplitude error also arises in the course of generating Ising gates on two exchange-coupled singlet-triplet qubits. ${ }^{16,22}$ We may, therefore, adopt an approach similar to what was used in that context and consider a generalisation of the SK1 composite pulse sequence: ${ }^{35}$

$$
\begin{aligned}
& e^{-i \sigma_{x} \otimes 1 \theta} \tilde{E}(N \pi, \delta \epsilon) e^{2 i \sigma_{x} \otimes I \theta} \tilde{E}(N \pi, \delta \epsilon) e^{-i \sigma_{x} \otimes l \theta} \tilde{E}(\phi, \delta \epsilon) \\
& =\tilde{E}(\phi, 0)\left[I \otimes I-i(2 N \pi \cos (2 \theta)+\phi)\left(\sigma_{z} \otimes \sigma_{z}\right) \delta \epsilon\right]+\mathcal{O}\left(\delta \epsilon^{2}\right) .
\end{aligned}
$$

If we choose $\theta= \pm \arccos (-\phi / 2 N \pi) / 2$, then the charge noise can be completely suppressed to the first order in $\delta \epsilon$. This sequence is shown as the quantum circuit in Figure 2a. An example of the gate infidelity as a function of the magnitude of charge noise is shown in Figure 2. The difference in slopes of the two curves signifies full cancelation of the first-order error, and one can see that for charge noise at the few-percent level, the error can be reduced by more than an order of magnitude.

\section{Reducing nuclear noise for nonzero $h$}

In actual experiments, one needs a nonzero $h$ to generate $x$-rotations, so we must depart from the ideal $h=0$ limit discussed above. In this case, the right-hand side of equation (7) is no longer an anti-diagonal matrix. As its explicit analytical form is complicated, one must check its equivalence to a CNOT gate by directly evaluating the Makhlin invariant. Taking the 'level-1' sequence, equation (8), as an example, the cost function now has a given nonzero $h$ value: $\mathcal{K}(J, \phi)=\|\partial \tilde{F}(J, h, \phi, \delta h) / \partial \delta h\|^{2}$, but most importantly, we have an extra constraint in that $\tilde{F}$ must be equivalent to CNOT. To impose this constraint, we define a function $\mathcal{M}(Q)=\left|G_{1}\right|^{2}+\left|G_{2}-1\right|^{2}$, where $Q$ is a $4 \times 4$ unitary, and $G_{1}, G_{2}$ are the Makhlin invariants associated with it. This function may be viewed as the 'distance' between the net evolution generated by any pulse sequence and the desired entangling gate.

Because $\phi=J^{A B} t$ no longer solely determines whether the gate is equivalent to CNOT, we will use $\phi$ as a search parameter in addition to $J$. Our optimisation problem thus becomes the minimisation of $\mathcal{K}(J, \phi)$ subject to the constraints $J \geqslant J^{A B}, \phi>0$ and the additional constraint from the Makhlin invariance $|\mathcal{M}(\tilde{F})| \leqslant \eta$, with $\eta$ a small dimensionless number, which we take to be $10^{-10}$ in our calculations. This additional constraint makes a brute-force numerical optimisation very difficult even if there are now more free parameters, because during the search, a given point in the parameter search space is only allowed to evolve in a very narrow strip determined by $\eta$. We, therefore, do not find such a brute-force direct strategy to be practical here. Instead, we adopt a more practical strategy in which we use the known result for $h=0$ and slowly turn on $h$, increasing it in small increments of size $\Delta h$ towards a desired value. This practice ensures that with 
each $h$ value, the search initiates from the solution for $h-\Delta h$, which is presumably not far away from the optimal solution for $h$, while at the same time satisfying the constraint of Makhlin invariance. This iterative discretized numerical strategy seems to be practical and should give the same answer as a direct numerical brute-force technique.

A typical result for $h=5 f^{A B}$ is shown in Figure 3. Figure 3a shows the quantum circuit used in this case, and Figure $3 \mathrm{~b}$ depicts the dependence of gate infidelity on the nuclear noise level. An orderof-magnitude reduction in error can be seen for a range of $\delta h / h$ around $10 \%$. In producing the gate infidelity, we convert the output of the sequence of equation (8) without noise to CNOT via

a

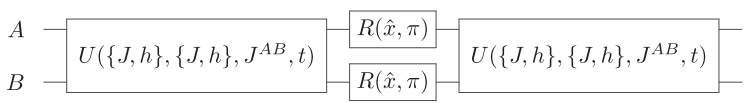

\section{b}

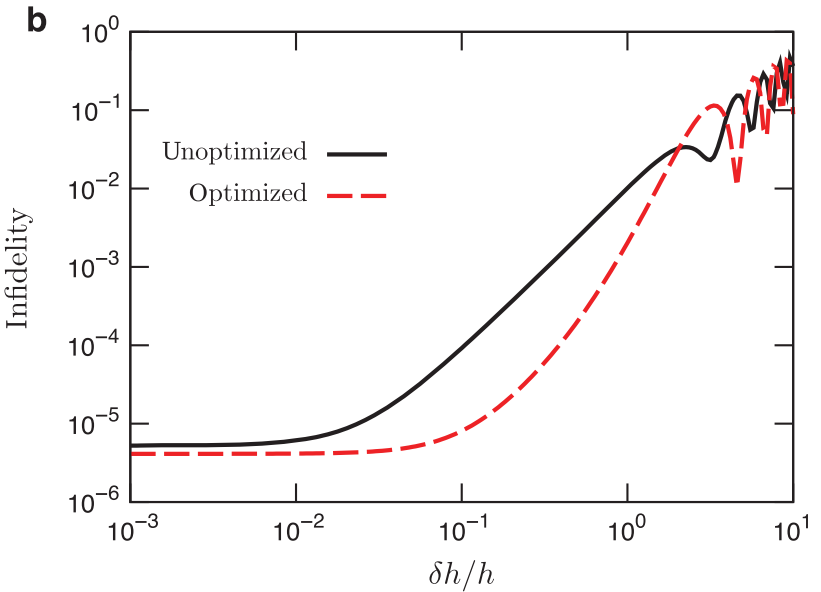

Figure 3. Nuclear noise reduction for $h=5 J^{A B}$. The charge noise has been set to zero. (a) Quantum circuit showing the pulse sequence used to reduce the nuclear noise. (b) Infidelity versus nuclear noise $\delta \mathrm{h} / \mathrm{h}$ for unoptimized (black line) and optimised (red dashed line) sequences. Parameters: unoptimized pulse: $J=124.83 J^{A B}$, $\phi=2 J^{A B} t=2.3601$. Optimised pulse: $J=134.20{ }^{A B}, \phi=2.3596$. Both the sequences are converted by (noiseless) local operations to CNOT to calculate the infidelity. local operations. Namely, we find real vectors $\boldsymbol{r}_{L}^{A}, \boldsymbol{r}_{L}^{B}, \boldsymbol{r}_{R^{\prime}}^{A}, \boldsymbol{r}_{R^{\prime}}^{B}$ such that

$$
\begin{aligned}
& \exp \left\{-i\left[\left(\boldsymbol{r}_{L}^{A} \cdot \boldsymbol{\sigma}\right) \otimes I+I \otimes\left(\boldsymbol{r}_{L}^{B} \cdot \boldsymbol{\sigma}\right)\right]\right\} \cdot Q \cdot \exp \left\{-i\left[\left(\boldsymbol{r}_{R}^{A} \cdot \boldsymbol{\sigma}\right) \otimes I+I \otimes\left(\boldsymbol{r}_{R}^{B} \cdot \boldsymbol{\sigma}\right)\right]\right\} \\
& \quad=\text { CNOT, }
\end{aligned}
$$

where $\boldsymbol{\sigma}=\left\{\sigma_{x}, \sigma_{y}, \sigma_{z}\right\}$. (The local operations are assumed to be free from noise.) As a consequence, the extent to which the entangling gate $Q$ resembles CNOT is manifest in Figure 3 as a nonzero saturation value for small $\delta h$. This error is on the order of $\sqrt{\eta}$, and as long as $\eta<10^{-8}$, it is small enough to not hinder quantum error correction. We also note that the $J$ values used for both unoptimized and optimised pulses in Figure 3 are larger compared with Figure 1. These larger values are required in the nonzero $h$ case to ensure that $Q$ is close enough to CNOT, i.e., within the prescribed precision set by $\eta$. Smaller $J$ may certainly be used at the cost of increasing $\eta$, and we have verified that solutions can be found for most cases as long as $\eta<10^{-6}$.

Simultaneous reduction of nuclear noise and charge noise for nonzero $h$

To make the sequence as robust as possible against both nuclear noise and charge noise, we first rewrite the longer sequence of equation (15) as

$$
\tilde{D}=e^{-2 i \sigma_{x} \otimes 1 \theta_{a}} F\left(J_{a}, h, \phi_{a}\right) e^{4 i \sigma_{x} \otimes 1 \theta_{b}} F\left(J_{b}, h, \phi_{b}\right) e^{-2 i \sigma_{x} \otimes 1 \theta_{c}} F\left(J_{c}, h, \phi_{c}\right),
$$

and we now have nine free parameters that can be tuned during the optimisation: $J_{a}, J_{b}, J_{c}, \phi_{a}, \phi_{b}, \phi_{c}, \theta_{a}, \theta_{b}$ and $\theta_{c}$. Again, our strategy is to start from the known solution at $h=0$ and then slowly turn on $h$ to ensure that the search always starts from a near-optimal solution while satisfying the constraint $|\mathcal{M}(\tilde{D})| \leqslant \eta$ as much as possible. In addition to the Makhlin invariant, other constraints include $J_{a, b, c} \geqslant J^{A B}, \phi_{a, b, c} \geqslant 0$. There are no constraints on $\theta_{a, b, c}$ as they denote exact single-qubit operations.

The cost function contains contributions from both the nuclear noise and the charge noise, but as they are not directly comparable, one must assign a weight $w$ when summing the two contributions in the cost function. We may, therefore, write

a
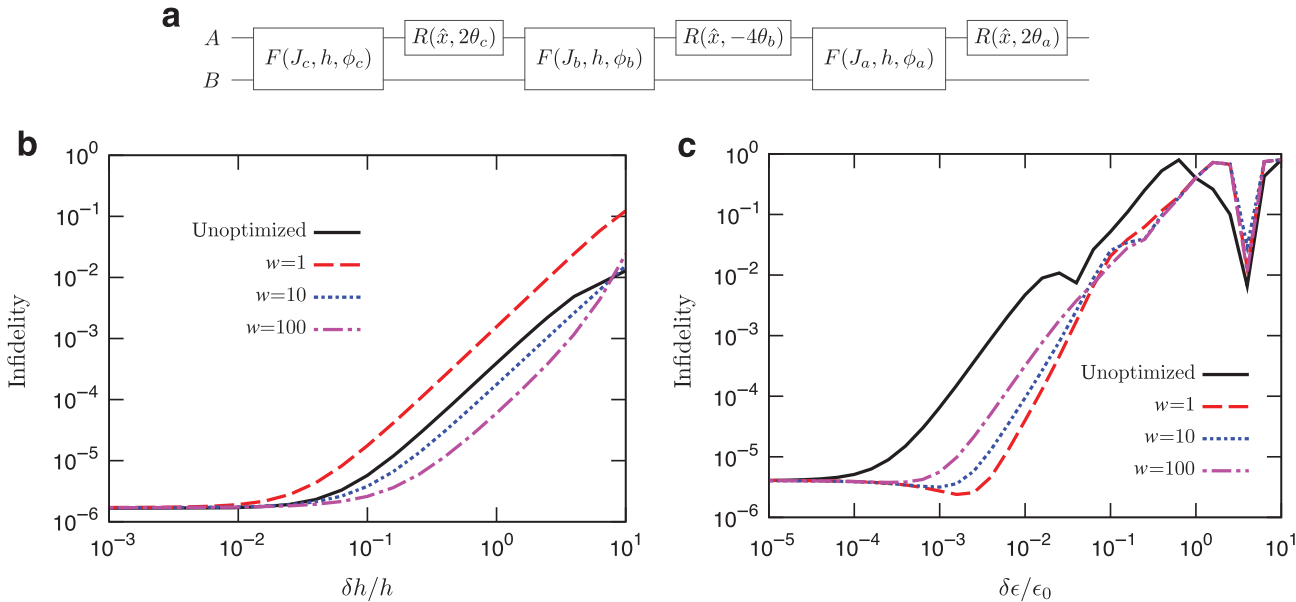

Figure 4. Simultaneous reduction of nuclear and charge noise for $h=2.4{ }^{A B}$. (a) Quantum circuit showing the pulse sequence used to reduce the noise. (b) Infidelity versus nuclear noise $\delta h / h$ with charge noise fixed at zero. (c) Infidelity versus charge noise $\delta \varepsilon / \varepsilon_{0}$ with zero nuclear noise. Parameters: unoptimized pulse: $J=64.128 J^{A B}, \phi=2.3596$. Optimised pulse with $w=1: J_{a}=151.56 J^{A B}, J_{b}=58.609 J^{A B}, J_{c}=33.712 J^{A B}, \phi_{a}=0.21612$, $\phi_{b}=0.35106, \phi_{c}=0.22303, \theta_{a}=14.200, \theta_{b}=1.5999$ and $\theta_{c}=3.0629$. Optimised pulse with $w=10: J_{a}=89.590 J^{A B}, J_{b}=83.522 J^{A B}, J_{c}=38.413 J^{A B}{ }^{\prime}$ $\phi_{a}=0.33514, \phi_{b}=0.27385, \phi_{c}=0.17992, \theta_{a}=22.690, \theta_{b}=1.5253$ and $\theta_{c}=3.0535$. Optimised pulse with $w=100: J_{a}=115.02 J^{A B^{\prime}}, J_{b}=64.424 J^{A B^{\prime}}$, $J_{c}=36.711 \mathrm{~J}^{A B}, \phi_{a}=0.24015, \phi_{b}=0.366112, \phi_{c}=0.18231, \theta_{a}=12.940, \theta_{b}=1.5308$ and $\theta_{c}=3.1414$. 
the cost function as

$$
\mathcal{K}=\left\|w \frac{\partial \tilde{D}}{\partial \delta h}\right\|^{2}+\left\|\frac{\partial \tilde{D}}{\partial \delta \varepsilon}\right\|^{2} .
$$

In practice, $w$ should be chosen according to whether charge noise or nuclear noise is responsible for most of the decoherence. Increasing $w$ means that one is willing to sacrifice a portion of the charge noise reduction in exchange for an additional reduction in nuclear noise, and vice versa. The results for several $w$ values are shown in Figure 4. Figure 4a shows the quantum circuit used. Figure $4 \mathrm{~b}$ shows the dependence of the infidelity on nuclear noise for zero charge noise, while Figure $4 c$ shows how the infidelity changes in accordance with the charge noise when the nuclear noise is absent. As in the previous section, we convert the resulting gate in the absence of noise to CNOT before evaluating the infidelities, so that the error resulting from the Makhlin constraint characterized by $\eta$ may be seen as part of the infidelities. For $w=1$, reduction of the charge noise is most pronounced, but the sequence offers no reduction in nuclear noise. The error actually increases from the unoptimized short sequence, due to the complexity of equation (17) compared with equation (8). As expected, when one increases $w$, the sequence starts to cancel nuclear noise, at the cost of offering less (but still acceptable) reduction in charge noise. For $w=100$, the sequence reduces both nuclear noise and charge noise by approximately one order of magnitude. The added flexibility afforded by $w$ is not only beneficial in experiments where one type of noise dominates over the other, but also in situations where one wishes to simultaneously employ alternative methods for suppressing noise, such as Bayesian estimation. ${ }^{14}$

\section{DISCUSSION}

The method presented here can be easily extended to generate entangling gates other than CNOT or applied to systems other than the singlet-triplet qubits discussed here. If one simply wishes to generate an arbitrary entangling gate in a noise-resistant fashion, then the constraint imposed by the Makhlin invariants can be removed, and it should be much easier to find optimal driving parameters. (The invariants must still be monitored to ensure that a non-entangling product of local gates is not generated.) Very recent experiments in double-quantum-dot charge qubits have demonstrated a CNOT gate with a fidelity comparable to that obtained for singlet-triplet spin qubits, ${ }^{36}$ and the coupling Hamiltonian is identical to equation (1) when $\rho^{A}, J^{B}$ are interpreted as the energy splittings between charge states and $h^{A}, h^{B}$ as the inter-dot tunnelings. In this case, the energy splittings are no longer constrained to be strictly positive, and their dependence on the detuning is typically linear. These differences can easily be incorporated into our optimisation procedure, and all the analysis presented here, including the structure of the pulse sequences, remains the same. We further note that our method may be generalised to produce noise-suppressing pulse sequences for other types of qubits, such as the exchange-only qubit or the resonant-exchange qubit, ${ }^{37-39}$ as well as the hybrid qubit demonstrated recently, 8,40 which can be coupled capacitively. We also emphasise that several assumptions that we have made for the purposes of presentation, including symmetric pulsing and the exponential charge noise model, can easily be lifted to address more general situations. For example, asymmetric pulsing may be treated by adding search parameters, while for a general charge noise model the sequence of equation (15) still holds as long as the auxiliary angle $\theta$ is determined by the actual amplitude errors of $\tilde{E}(N \pi, \delta \varepsilon)$ and $\tilde{E}(\phi, \delta \varepsilon)$. Discussions for similar cases in exchange-coupled qubits may be found in reference 22. Reference 22 also treats a case where the static-noise approximation is lifted, and has found that as long as the noise is concentrated at low frequencies, dynamically corrected singlequbit gates work fine. In future work, it would be interesting to map out precisely the types of noise spectra for which our twoqubit gates do and do not work well, although it will be computationally much more expensive than in the case of singlequbit gates.

Precise, error-free entangling gate operations on coupled qubits is currently the bottleneck for scalable quantum computation using solid-state spin qubits. Our results show how to reduce both nuclear noise and charge noise while at the same time offering the advantage that the sequences are simple enough for immediate experimental implementation. The simpler sequence for nuclear noise reduction in the case of negligible Overhauser field gradients only requires tuning the value of the exchange coupling in the existing experimental control protocol, ${ }^{19}$ while our more powerful sequence that suppresses both types of noise extends that sequence merely by a factor of three. We, therefore, believe that our results are of immediate practical use and constitute an important step toward scalable semiconductor quantum technologies on the basis of coupled spin qubits.

\section{ACKNOWLEDGEMENTS}

This work is supported by LPS-CMTC and IARPA-MQCO grants.

\section{COMPETING INTERESTS}

The authors declare no conflict of interest.

\section{REFERENCES}

1 Nielsen M, Chuang I. Quantum Computation and Quantum Information. Cambridge University Press: Cambridge, UK, 2000.

2 DiCarlo L, Chow JM, Gambetta JM, Bishop LS, Johnson BR, Schuster DI et al. Demonstration of two-qubit algorithms with a superconducting quantum processor. Nature 2009; 460: 240-244.

3 Gulde S, Riebe M, Lancaster GPT, Becher C, Eschner J, Haffner H et al. Implementation of the Deutsch-Jozsa algorithm on an ion-trap quantum computer. Nature 2003; 421: 48-50.

4 O'Brien JL, Pryde GJ, White AG, Ralph TC, Branning D. Demonstration of an all-optical quantum controlled-not gate. Nature 2003; 426: 264-267.

5 Nowack KC, Shafiei M, Laforest M, Prawiroatmodjo GEDK, Schreiber LR, Reichl C et al. Single-shot correlations and two-qubit gate of solid-state spins. Science 2011; 333: 1269-1272.

6 Brunner R, Shin Y-S, Obata T, Pioro-Ladrière M, Kubo T, Yoshida K et al. Two-qubit gate of combined single-spin rotation and interdot spin exchange in a double quantum dot. Phys Rev Lett 2011; 107: 146801.

7 Veldhorst M, Yang CH, Hwang JCC, Huang W, Dehollain JP, Muhonen JT et al. A two qubit logic gate in silicon. Nature 2015; 526: 410-414. doi:10.1038/nature15263.

8 Kim D, Shi Z, Simmons CB, Ward DR, Prance JR, Koh TS et al. Quantum control and process tomography of a semiconductor quantum dot hybrid qubit. Nature 2014; 511: 70-74.

9 Muhonen JT, Dehollain JP, Laucht A, Hudson FE, Kalra R, Sekiguchi T et al. Storing quantum information for 30 seconds in a nanoelectronic device. Nat Nanotechnol 2014; 9: 986-991.

10 Taylor JM, Engel HA, Dur W, Yacoby A, Marcus CM, Zoller P et al. Fault-tolerant architecture for quantum computation using electrically controlled semiconductor spins. Nat Phys 2005; 1: 177-183.

11 Petta J, Johnson A, Taylor J, Laird E, Yacoby A, Lukin M et al. Coherent manipulation of coupled electron spins in semiconductor quantum dots. Science 2005; 309: 2180-2184.

12 Foletti S, Bluhm H, Mahalu D, Umansky V, Yacoby A. Universal quantum control of two-electron spin quantum bits using dynamic nuclear polarization. Nat Phys 2009; 5: 903-908.

13 Bluhm H, Foletti S, Neder I, Rudner M, Mahalu D, Umansky V et al. Dephasing time of GaAs electron-spin qubits coupled to a nuclear bath exceeding $200 \mu \mathrm{s}$. Nat Phys 2011; 7: 109-113.

14 Shulman MD, Harvey SP, Nichol JM, Bartlett SD, Doherty AC, Umansky V et al. Suppressing qubit dephasing using real-time Hamiltonian estimation. Nat Commun 2014; 5: 5156.

15 Klinovaja J, Stepanenko D, Halperin B, Loss D. Exchange-based CNOT gates for singlet-triplet qubits with spin-orbit interaction. Phys Rev B 2012; 86: 085423. 
16 Kestner JP, Wang X, Bishop LS, Barnes E, Das Sarma S. Noise-resistant control for a spin qubit array. Phys Rev Lett 2013; 110: 140502.

17 van Weperen I, Armstrong B, Laird E, Medford J, Marcus C, Hanson M et al. Chargestate conditional operation of a spin qubit. Phys Rev Lett 2011; 107: 030506

18 Trifunovic L, Dial O, Trif M, Wootton J, Abebe R, Yacoby A et al. Long-distance spin-spin coupling via floating gates. Phys Rev $X$ 2012; 2: 011006.

19 Shulman MD, Dial OE, Harvey SP, Bluhm H, Umansky V, Yacoby A. Demonstration of entanglement of electrostatically coupled singlet-triplet qubits. Science 2012; 336: 202-205.

20 Wang X, Bishop LS, Kestner JP, Barnes E, Sun K, Das Sarma S. Composite pulses for robust universal control of singlet-triplet qubits. Nat Commun 2012; 3: 997.

21 Grace M, Dominy J, Witzel W, Carroll M. Optimized pulses for the control of uncertain qubits. Phys Rev A 2012; 85: 052313.

22 Wang X, Bishop LS, Barnes E, Kestner JP, Sarma SD. Robust quantum gates for singlet-triplet spin qubits using composite pulses. Phys Rev A 2014; 89: 022310.

23 Barnes E, Wang X, Das Sarma S. Robust quantum control using smooth pulses and topological winding. Sci Rep 2015; 5: 12685. doi:10.1038/srep12685.

24 Jones JA. Robust ising gates for practical quantum computation. Phys Rev A 2003; 67: 012317.

25 Calderon-Vargas FA, Kestner JP. Directly accessible entangling gates for capacitively coupled singlet-triplet qubits. Phys Rev B 2015; 91: 035301.

26 Srinivasa V, Taylor JM. Capacitively coupled singlet-triplet qubits in the double charge resonant regime, 2014. Preprint at $\langle$ http://arxiv.org/abs/1408.4740 .

27 Johnson AC, Petta JR, Taylor JM, Yacoby A, Lukin MD, Marcus CM et al. Triplet-singlet spin relaxation via nuclei in a double quantum dot. Nature 2005; 435: 925-928.

28 Medford J, Cywiński L, Barthel C, Marcus C, Hanson M, Gossard A. Scaling of dynamical decoupling for spin qubits. Phys Rev Lett 2012; 108: 086802.

29 Reilly DJ, Taylor JM, Laird EA, Petta JR, Marcus CM, Hanson MP et al. Measurement of temporal correlations of the Overhauser field in a double quantum dot. Phys Rev Lett 2008; 101: 236803.

30 Dial O, Shulman M, Harvey S, Bluhm H, Umansky V, Yacoby A. Charge noise spectroscopy using coherent exchange oscillations in a singlet-triplet qubit. Phys Rev Lett 2013; 110: 146804.
31 Maune BM, Borselli MG, Huang B, Ladd TD, Deelman PW, Holabird KS et al. Coherent singlet-triplet oscillations in a silicon-based double quantum dot. Nature 2012; 481: 344-347

32 Wu X, Ward DR, Prance JR, Kim D, Gamble JK, Mohr RT et al. Two-axis control of a singlet-triplet qubit with an integrated micromagnet. Proc Natl Acad Sci USA 2014; 111: 11938-11942.

33 Wang X, Calderon-Vargas FA, Rana MS, Kestner JP, Barnes E, Das Sarma S. Noise-compensating pulses for electrostatically controlled silicon spin qubits. Phys Rev B 2014; 90: 155306

34 Makhlin Y. Nonlocal properties of two-qubit gates and mixed states, and the optimization of quantum computations. Quant Inf Proc 2002; 1: 243-252.

35 Brown K, Harrow A, Chuang I. Arbitrarily accurate composite pulse sequences. Phys Rev A 2004; 70: 052318

36 Li H-O, Cao G, Yu G-D, Xiao M, Guo G-C, Jiang H-W et al. Controlled-NOT quantum logic gate in two strongly coupled semiconductor charge qubits. Nat Commun 2015; 6: 7681. doi:10.1038/ncomms8681.

37 DiVincenzo DP, Bacon D, Kempe J, Burkard G, Whaley KB. Universal quantum computation with the exchange interaction. Nature 2000; 408: 339-342.

38 Medford J, Beil J, Taylor J, Rashba E, Lu H, Gossard A et al. Quantum-dot-based resonant exchange qubit. Phys Rev Lett 2013; 111: 050501.

39 Pal A, Rashba El, Halperin B I. Driven nonlinear dynamics of two coupled exchange-only qubits. Phys Rev X 2014; 4: 011012.

40 Shi Z, Simmons C, Prance J, Gamble J, Koh T, Shim Y-P et al. Fast hybrid silicon double-quantum-dot qubit. Phys Rev Lett 2012; 108: 140503.

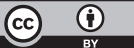

This work is licensed under a Creative Commons Attribution 4.0 International License. The images or other third party material in this article are included in the article's Creative Commons license, unless indicated otherwise in the credit line; if the material is not included under the Creative Commons license, users will need to obtain permission from the license holder to reproduce the material. To view a copy of this license, visit http://creativecommons.org/licenses/ by/4.0/ 